\title{
Dentin Dysplasia Type I: A Rare Case Report and Management Protocol
}

\author{
Shimasadat Miri ${ }^{1}$, Mahsasadat Miri ${ }^{2}$, Parisa Soltani ${ }^{3, *}$, Amirhossein Moaddabi $^{4}$ \\ ${ }^{1}$ Department of Endodontics, School of Dentistry, Kermanshah University of Medical Sciences, Kermanshah, Iran \\ ${ }^{2}$ Department of Obstetrics and Gynecology, School of Medicine, Isfahan University of Medical Sciences, Isfahan, Iran \\ ${ }^{3}$ Department of Oral and Maxillofacial Radiology, School of Dentistry, Isfahan University of Medical Sciences, Isfahan, Iran \\ ${ }^{4}$ Department of Oral and Maxillofacial Surgery, School of Dentistry, Mazandaran University of Medical Sciences, Sari, Iran \\ *Corresponding author: parisa.soltani@live.com
}

\begin{abstract}
Dentin Dysplasia (DD) is a rare autosomal dominant anomaly that disturbs the formation of dentin in primary and/or permanent dentitions. This condition is classified into types I (radicular) and II (coronal) based on radiological findings. A case of DD type I in an 11-year old Iranian boy is presented and the clinical and radiological findings and selected treatment plan are discussed.
\end{abstract}

Keywords: dentin dysplasia, radicular, pediatric dentistry

Cite This Article: Shimasadat Miri, Mahsasadat Miri², Parisa Soltani, and Amirhossein Moaddabi, "Dentin Dysplasia Type I: A Rare Case Report and Management Protocol." International Journal of Dental Sciences and Research, vol. 5, no. 5 (2017): 122-124. doi: 10.12691/ijdsr-5-5-2.

\section{Introduction}

Dentin Dysplasia (DD) is a rare autosomal dominant anomaly that disturbs the formation of dentin either in the primary or both primary and permanent dentitions. Prevalence of this condition is reported to be approximately 1 in 100,000 patients [1]. Ballschmiede was the first one reporting this malformation as 'rootless teeth' in 1920 [2]. In 1939 Rushton suggested the term DD for this condition [3]. In 1972 Witkop classified DD into two types based on radiological findings: type I (DDI: radicular dysplasia) and type II (DDII: coronal dysplasia) [4].

DDI is characterized by presence of primary and permanent teeth with morphologically normal or slightly amber colored crown with no or only rudimentary root development, improper growth of dentin resulting in incomplete or total obliteration of the pulp chamber, and periapical radiolucent areas or cysts which can lead to early loss of tooth $[5,6,7]$. Although delayed eruption of teeth is reported in DDI, generally teeth erupt in the normal time [8]. In DDI teeth appear to have greater resistance to caries than normal teeth [9]. In histologic examination, the enamel and immediately subjacent dentin appear normal. Deeper layers of dentin show atypical tubular patterns with amorphous, atubular areas and irregular organization. Globular or nodular masses of abnormal dentin are seen pulpally to the normal appearing dentin [10].

The present report describes a case of DDI in an 11-year-old boy attending a private office in Iran.

\section{Case Report}

An 11-year-old boy attended to a private office for dental examination. Extraoral examination and medical history did not indicate any abnormal finding. Intraoral examination revealed poor oral hygiene of the patient, class I occlusion, and gingivitis. The teeth did not show pathological mobility or infection or abnormal appearance. Figure 1 depicts the intraoral photograph of the patient. Teeth number 15 and 31 had caries and the upper canines were not completely erupted. Radiographic examinations revealed no cystic or radiolucent lesion. Panoramic and intraoral radiographs of the case are presented in Figure 2. All teeth had short and malformed roots with closed pulp chambers. The patient was diagnosed with DDI based on clinical and radiologic examinations. This condition was not observed in other family members of the patient.

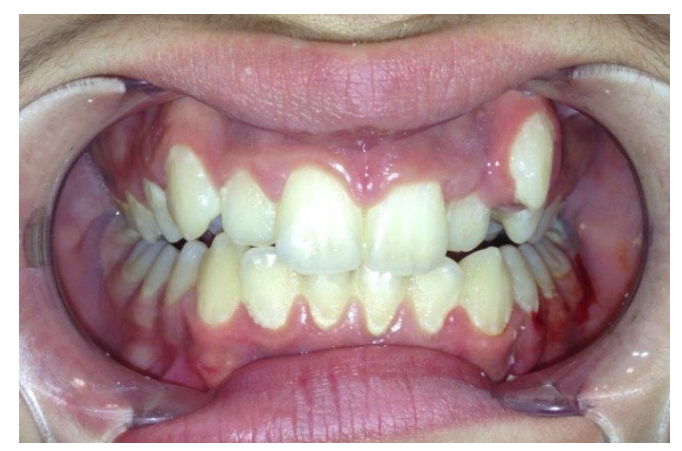

Figure 1. Intraoral photograph of the patient 


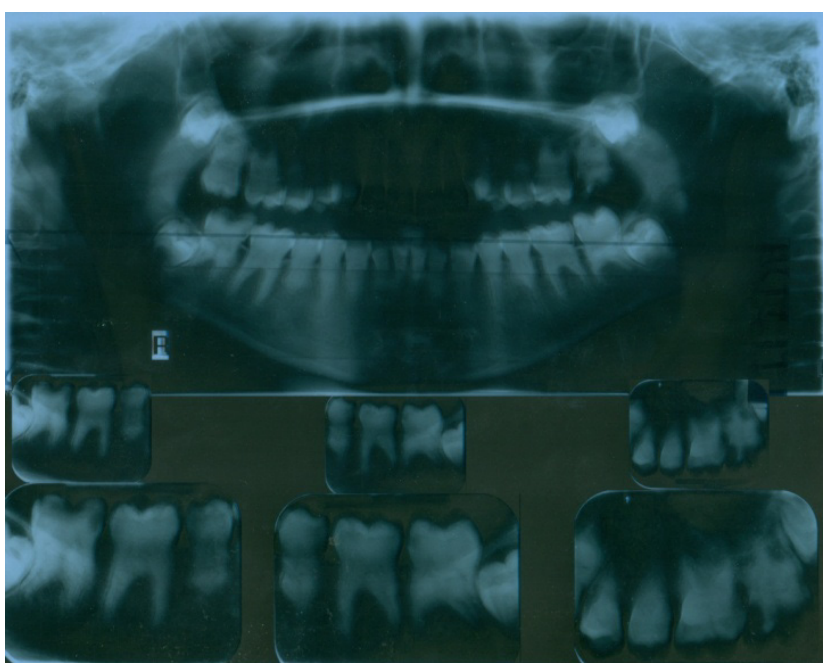

Figure 2. Panoramic and intraoral radiographs of the patient

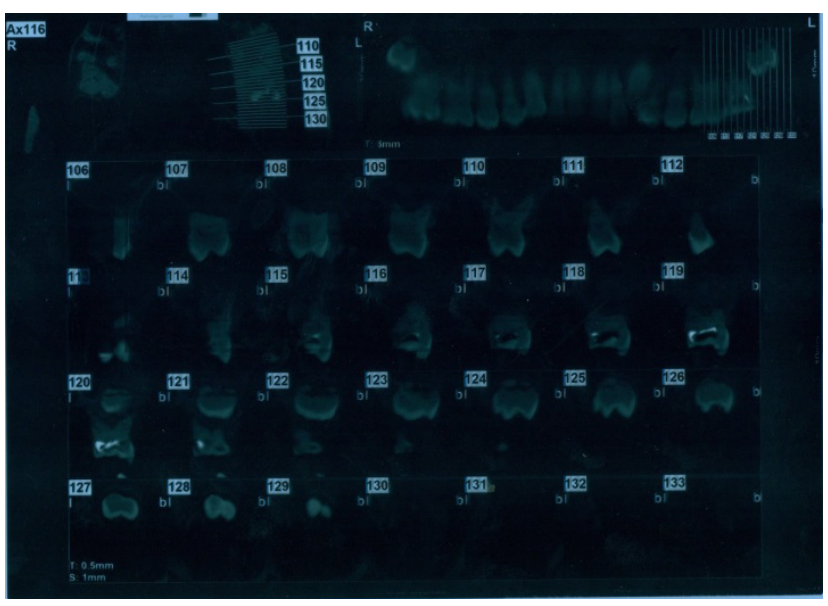

Figure 3. Cone-beam computed tomography of maxilla

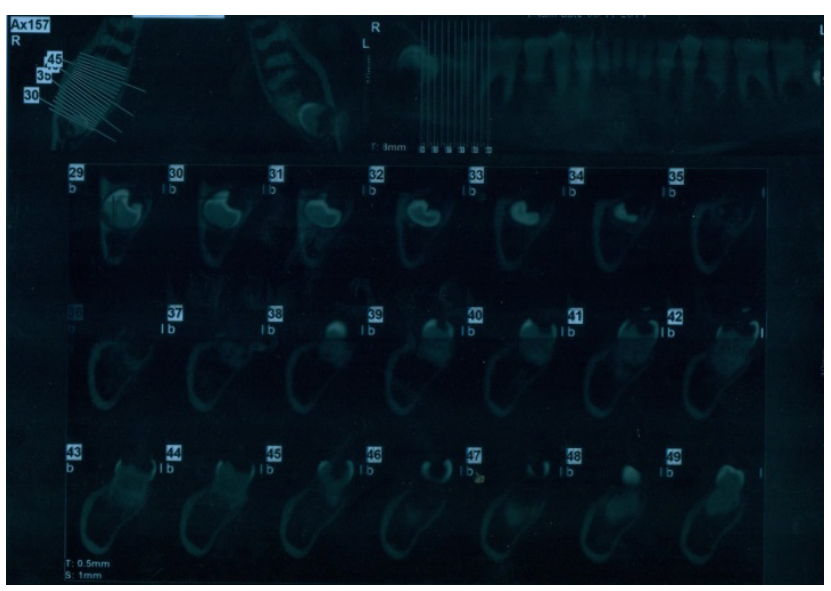

Figure 4. Cone-beam computed tomography of mandible

Treatment was planned based on oral hygiene improvement and restoration of caries. Due to deep caries in tooth number 15 and exposure of the pulp horn, access cavity was prepared. But as the pulp chamber was obliterated and root canal therapy was not possible, the access cavity was sealed by MTA and the tooth was restored. Afterwards, the patient was followed for about 1 year and no further complication was observed. Figure 3 and Figure 4 show cone-beam computed tomography of maxilla and mandible, respectively.

\section{Discussion}

As mentioned DD is a disturbance in dentin formation. The etiological and pathogenesis basis of DD is not exactly determined yet. Extracellular matrix of the dentin is the result of the differentiation of neural crest ectomesenchymal cells into odontoblasts. Odontoblasts express specific genes products which form the collagenous dentin extracellular matrix. This matrix consists of mostly type I, type I trimer, type III, type V, and type VI collagens and several noncollagenous proteins also found in bone extracellular matrix, such as osteonectin, osteocalcin, osteopontin, bone sialoprotein, and dentin matrix protein 1 . However, two dentin matrix proteins, dentin sialoprotein (DSP) and dentin phosphoprotein (DPP), are expressed by odontoblasts and transiently by ameloblasts [11]. These noncollagenous proteins are believed to be essential for initiation and control of mineralization in the transition of predentin to dentin [12]. DSP and DPP are cleavage products expressed from a single transcript encoded by dentin sialophosphoprotein (DSPP) gene on human chromosome 4 [13]. Mutations in this gene are detected in several disorders of dentin formation process including DD and dentinogenesis imperfecta $[14,15,16]$. Wesley et al suggested that abnormal interactions of odontoblasts with ameloblasts resulting in abnormal differentiation and function of odontoblasts can cause DD [17]. Logan et al commented that multiple degenerative foci in the dental papilla become calcified and it can cause obliteration and reduced growth of the pulp space [18]. However Sauk et al proposed that early invagination of the epithelial root sheath can induce ectopic dentin formation in the pulp space and can obliterate the pulp chamber [19].

Coronal morphology in DDI is usually normal $[5,20,21]$ In our case the teeth had normal size and shape but were slightly amber colored. Moreover, malocclusion is not a striking feature in DDI [22]. However, it can be present in some cases. Our patient had Class 1 occlusion with posterior open bite.

Management of DD patients can be problematic in some cases. Follow up and routine conservative treatment is the desired treatment plan in DD cases [23]. Extraction of infected teeth with periapical abscess can be considered as the final choice [24]. Teeth with relatively long roots can undergo endodontic treatment [9] if the pulp chamber is not obliterated. Early exfoliation of teeth and associated bone loss can necessitate techniques such as onlay bone grafting and sinus lift for placement of dental implants in adults [25]. In our case oral hygiene improvement was suggested to prevent from further dental complications. Restoration of teeth with carious lesions was considered. As endodontic treatment of tooth number 15 was not possible due to pulpal obliteration, it was sealed with MTA and restored. One-year follow-up revealed no complications in this case.

\section{Conclusion}

Dentin dysplasia type $I$ is a rare genetic anomaly in dentin formation which can lead to early exfoliation of 
primary and/or permanent dentition. Early diagnosis and preventive measures to avoid further complications is of considerable importance in management of patients with DDI.

\section{References}

[1] Kim JW, Simmer JP. Hereditary dentin defects. Journal of dental research. 2007; 86(5): 392-9.

[2] Herbst E, Apffelstaedt M. Malformations of the Jaws and Teeth: Oxford University Press; 1930.

[3] Rushton MA. A case of dentinal dysplasia. Guys Hosp Rep. 1939; 89(89): 369-73.

[4] Witkop Jr CJ. Hereditary defects of dentin. Dent Clin North Am 1975; 19(1): 25-45.

[5] Carroll MKO, Duncan WK. Dentin dysplasia type I: Radiologic and genetic perspectives in a six-generation family. Oral Surg Oral Med Oral Pathol. 1994; 78(3): 375-81.

[6] Depprich RA, Ommerborn MA, Handschel JG, Naujoks CD, Meyer U, Kübler NR. Dentin dysplasia type I: a challenge for treatment with dental implants. Head Face Med. 2007; 3(1): 1.

[7] Barron MJ, McDonnell ST, MacKie I, Dixon MJ. Hereditary dentine disorders: dentinogenesis imperfecta and dentine dysplasia. Orphanet J Rare Dis. 2008; 3(1): 1.

[8] Scola SM, Watts PG. Dentinal dysplasia type I. A subclassification. Br J Orthod. 1987; 14(3): 175-9.

[9] Tidwell E, Cunningham CJ. Dentinal dysplasia: endodontic treatment, with case report. J Endod. 1979; 5(12): 372-6.

[10] Shields E, Bixler D, El-Kafrawy A. A proposed classification for heritable human dentine defects with a description of a new entity. Arch Oral Biol. 1973; 18(4): 543IN7-53.

[11] Butler WT, Ritchie $H$. The nature and functional significance of dentin extracellular matrix proteins. The International journal of developmental biology. 1995; 39(1): 169-79.

[12] Feng JQ, Luan X, Wallace J, Jing D, Ohshima T, Kulkarni AB, et al. Genomic organization, chromosomal mapping, and promoter analysis of the mouse dentin sialophosphoprotein (Dspp) gene, which codes for both dentin sialoprotein and dentin phosphoprotein. Journal of Biological Chemistry. 1998; 273(16): 9457-64.

[13] Gu K, Chang S, Ritchie HH, Clarkson BH, Rutherford RB. Molecular cloning of a human dentin sialophosphoprotein gene. European journal of oral sciences. 2000; 108(1): 35-42.

[14] Kim J-W, Nam S-H, Jang K-T, Lee S-H, Kim C-C, Hahn S-H, et al. A novel splice acceptor mutation in the DSPP gene causing dentinogenesis imperfecta type II. Human genetics. 2004; 115(3): 248-54.

[15] Xiao S, Yu C, Chou X, Yuan W, Wang Y, Bu L, et al. Dentinogenesis imperfecta 1 with or without progressive hearing loss is associated with distinct mutations in DSPP. Nature genetics. 2001; 27(2): 201-4.

[16] McKnight DA, Simmer JP, Hart PS, Hart TC, Fisher LW. Overlapping DSPP Mutations Cause Dentin Dysplasia and Dentinogenesis Imperfecta. Journal of dental research. 2008; 87(12): 1108-11.

[17] Wesley RK, Wysocki GP, Mintz SM, Jackson J. Dentin dysplasia type I: Clinical, morphologic, and genetic studies of a case. Oral Surg Oral Med Oral Pathol. 1976; 41(4): 516-24.

[18] Logan J, Becks H, Silverman S, Pindborg JJ. Dentinal dysplasia. Oral Surg Oral Med Oral Pathol. 1962; 15(3): 317-33.

[19] Sauk JJ, Lyon HW, Trowbridge HO, Witkop CJ. An electron optic analysis and explanation for the etiology of dentinal dysplasia. Oral Surg Oral Med Oral Pathol. 1972; 33(5): 763-71.

[20] Vieira AR, Modesto A, Cabral MG. Dentinal dysplasia type I: report of an atypical case in the primary dentition. ASDC J Dent Child. 1998; 65(2): 141.

[21] Kalk WW, Batenburg RH, Vissink A. Dentin dysplasia type I: five cases within one family. Oral Surg Oral Med Oral Pathol Oral Radiol Endod. 1998; 86(2): 175-8.

[22] Luffingham J, Noble H. Dentinal dysplasia. Br Dent J. 1986; 160(8): 281.

[23] Steidler N, Radden B, Reade P. Dentinal dysplasia: a clinicopathological study of eight cases and review of the literature. Br J Oral Maxillofac Surg. 1984; 22(4): 274-86.

[24] Shafer WG, MK Levy B. A textbook of oral pathology. 1983.

[25] Muñoz-Guerra MF, Naval-Gías L, Escorial V, Sastre-Pérez J. Dentin dysplasia type I treated with onlay bone grafting, sinus augmentation, and osseointegrated implants. Implant Dent. 2006; 15(3): $248-53$. 